\title{
Exploring Remote Learning Methods for User Training in Research Computing
}

\author{
Dhruva K. Chakravorty ${ }^{1}$ \\ chakravorty@tamu.edu \\ Braden Yosko ${ }^{1}$ \\ byosko@tamu.edu \\ Stuti H. Trivedi ${ }^{1}$ \\ stutitrivedi1373@tamu.edu
}

\author{
Lisa M. Perez ${ }^{1}$ \\ perez@tamu.edu \\ Keith Jackson ${ }^{1}$ \\ kjackson@tamu.edu \\ Levi Jordan ${ }^{1}$ \\ ljordan56@tamu.edu
}

\author{
Honggao Liu ${ }^{1}$ \\ honggao@tamu.edu \\ Dylan Rodriguez ${ }^{1}$ \\ dylan@tamu.edu \\ Shaina Le ${ }^{1}$ \\ sle1019@tamu.edu
}

\begin{abstract}
The COVID-19 national health crisis forced a sudden and drastic move to online delivery of instruction across the nation. This almost instantaneous transition from a predominantly traditional "inperson" instruction model to a predominantly online model has forced programs to rethink instructional approaches. Before COVID-19 and mandatory social distancing, online training in research computing (RC) was typically limited to "live-streaming" informal in-person training sessions. These sessions were augmented with hands-on exercises on live notebooks for remote participants, with almost no assessment of student learning. Unlike select instances that focused on an international audience, local training curricula were designed with the in-person attendee in mind. Sustained training for RC became more important since when several other avenues of research were diminished. Here we report on two educational approaches that were implemented in the informal program hosted by Texas A\&M High Performance Research Computing (HPRC) in the Spring, Summer, and Fall semesters of 2020. These sessions were offered over Zoom, with the instructor assisted by moderators using the chat features. The first approach duplicated our traditional in-person sessions in an online setting. These sessions were taught by staff, and the focus was on offering a lot of information. A second approach focused on engaging learners via shorter pop-up courses in which participants chose the topic matter. This approach implemented a peer-learning environment, in which students taught and moderated the training sessions. These sessions were supplemented with YouTube videos and continued engagement over a community Slack workspace. An analysis of these approaches is presented.
\end{abstract}

\section{CCS CONCEPTS}

$\cdot \mathbf{C S} \rightarrow$ Computer Science; $\cdot$ Cybertraining $\rightarrow$ training on using cyberinfrastructure; $\cdot \mathbf{H P C} \rightarrow$ high performance computing

\footnotetext{
${ }^{1}$ High Performance Research Computing, Texas A\&M University, College Station, TX

Permission to make digital or hard copies of all or part of this work for personal or classroom use is granted without fee provided that copies are not made or distributed for profit or commercial advantage and that copies bear this notice and the full citation on the first page. To copy otherwise, or republish, to post on servers or to redistribute to lists, requires prior specific permission and/or a fee. Copyright CJOCSE, a supported publication of the Shodor Education Foundation Inc.
}

(C) 2021 Journal of Computational Science Education DOI: https://doi.org/10.22369/issn.2153-4136/12/2/2

\section{Keywords}

Online education, COVID-19, YouTube education, Cybertraining

\section{INTRODUCTION}

The COVID-19 national health crisis forced a sudden and drastic move to online delivery of instruction across the nation. This almost instantaneous transition from a predominantly traditional "inperson" instruction model to a predominantly online model has forced programs to rethink instructional approaches. Unlike select instances, such as the Petascale Institute, that have traditionally focused on a geographically-distributed audience, local campus computing training curricula were primarily designed with the inperson learner in mind. Prior to the changes brought by COVID 19related national social distancing norms, online training in research computing (RC) was typically limited to "live-streaming" informal in-person training sessions. For example, training and educational sessions offered by Texas A\&M HPRC [1] primarily focused on the "in-person" participants, with tracking, support, and strong assessments. The online experience was augmented with hands-on exercises on live notebooks for remote participants, with limited assessments of efficacy and student learning.

The impact of these adopted social norms affected research computing as well. In the Spring months of 2020, with a view toward combatting the spread of COVID-19, several institutions staggered, limited, or closed research facilities that required inperson interactions. While researchers were asked to practice social distancing at some institutions, at others they were encouraged to stay off campuses. Unable to perform physical experiments, computationally-curious, albeit untrained, researchers flocked to campus RC sites. For example, at Texas A\&M HPRC, we saw a significant increase in both new users and the number of job submissions on our clusters. This influx of new researchers offered opportunities to experiment with sustainable and scalable training approaches for researchers new to RC.

\section{ONLINE TRAINING AND EDUCATION}

Much like other campus research computing efforts, Texas A\&M HPRC has offered a series of training, outreach, and educational efforts that supports our researcher community [2-6]. Our user training program has been operational for several years, with thousands of participants signing up for events. At its heart are twoand-a-half-hour sessions, called the short course program, that are built along the idea of active-learning approaches [7-10]. Prior to March 2020, these sessions were offered both in-person and over live remote (Zoom/WebEx) modalities. These sessions were augmented with day-long workshops that were traditionally 
focused on in-person attendees. Both the short courses and workshops largely relied on our production environments Jupyter notebooks, virtual machines, and command line interface (CLI). While instructional aids (slide decks and Jupyter notebooks) were available on our website, video recordings of the courses were not available. These courses have been included in several formal curricular efforts at Texas A\&M. A detailed report on them has been presented elsewhere [2-6].

In response to social distancing policies recommended due to the COVID pandemic, we realized that we had to change our approach toward user training. We adopted two distinct approaches toward user training. For the Summer and Fall 2020 semesters, Texas A\&M HPRC chose to offer both versions of its informal learning program in an online modality. These programs continue to evolve as we experiment with pedagogical approaches to strengthen our curricula. Here we report on the progress, strengths, weaknesses, and opportunities to improve on these approaches. At the outset, these programs were offered over Zoom, with the instructor assisted by moderators using the chat features.

\subsection{Short Courses}

The first approach, called shortcourses, closely mirrored our traditional in-person focused sessions, albeit in an online-only setting. By design, these courses are detailed, informationintensive, and built as information resources that can be revisited by participating students. These are typically taught by experienced HPRC staff, Texas A\&M faculty, or scientists. Curricular materials are available for download from Github or the HPRC website. These courses are two-and-a-half hours long and are tiered with other short courses. To further establish a learning structure, these courses are often combined with complementary offerings, such as workshops or user group meetings. In the now online format, the course instructor was supported by other HPRC scientists over Zoom chat. The goal here is to offer a deeper introductory dive into computing. These courses are built on a tiered instruction model where the topics covered during the courses build on each other. As such, a learner can participate in courses throughout a semester and develop a comprehensive understanding of RC software and tools. To enable effective delivery, we developed a document describing expectations from presenters and participants on Zoom. To collect participant feedback on our courses, we migrated our surveys to Google Forms.

\subsection{Primers}

Toward the end of the Spring 2020 semester, we realized that our now online short course program was probably competing with other online commitments for a learner's time. We were also concerned that the short course program took a considerable amount of staff time away from responding to our growing userneeds. We also realized that, traditionally, new users often belonged to research groups that had roots in RC. In this scenario, we could rely on existing computing expertise within the new user's group to bring him/her/them up to speed. Due to the COVID19 crisis, we had a new set of researchers join research computing. These computationally-curious researchers belonged to research groups (or facilities) that didn't provide the scaffolding that our short course program relied on. Since these researchers came from varied backgrounds, we also didn't have a pre-existing framework that informed us what and how these researchers wanted to learn.

Despite the curricular strengths of our short course program, we felt the need for a new pedagogical approach that taught the new generation of users while focusing on learner engagement [11]. Admiring the success of short videos on social media platforms such as TikTok and YouTube, we realized that online informal education could be offered as bursts of information rather than relying on a structured tiered learning approach. In a related vein, platforms such as Discord have successfully coupled "live streaming" with "live chat" to engage the audience. Here, the presenter performs a task and converses using the video feed, while the audience participates in a "live chat" where they react or add to the presenter's actions. During this time, we also noticed that users were requesting information via our Helpdesk ticketing system that could be scaled out via informational videos. These requests were typically handled by our experienced student technician group that includes members from current and previous Super Computing Student Cluster competition teams.

\section{EXPERIMENTAL DESIGN}

Driven by the need to innovate, and inspired by the opportunities in our operations group, we developed a second approach that focused on high learner engagement by offering information on demand. This program, called Primers, relied on 60-minute courses and moved away from the focus on a semester-long learning experience. The Primer courses were intended to provide a burst of information for learners in an online-learning-friendly format. For the Primers, we first identified core competencies that RC researchers need to know. These core competencies were identified via discussions with HPRC staff, consultation with groups working in this area, HPRC user tickers, our "Introduction to HPRC" short course, and its corresponding assessments.

As part of this design, we took a cue from pop-up courses and crowd sourced when and how often these topics should be taught. Towards this, the registration form allowed participants to vote on the courses that would be offered, the sub-topics to be covered during the course, and suggestions on what should be taught. As a rule, we required that a minimum of five learners had to register for a Primer course for it to be offered. The program was geared to offer quick information and get a user to actively work on the problem. Unlike our short course program, it had no explicit tiered or prolonged learning structure. As such, we anticipated learners signing up for one-off courses, with the learning limited to a single semester. Building on the depth of expertise in our student technician program, we implemented a peer-learning environment in which two experienced undergraduate or graduate students taught and moderated the training sessions.

Instructional materials for the Primers were prepared by Texas A\&M HPRC staff and students. While one student technician presented the material and guided the class through the hands-on sessions, the second student technician posted comments on Zoom chat and added additional information. Scaffolding was offered via materials like Jupyter notebooks [12]. Each 60-minute session was followed by a 15-minute informal "Open Mic" session during which, participants could chat or talk about any topic related to RC. To ensure success, we endeavored to build a support structure along the live courses. To capture these discussions and foster closer collaborations among researchers, participants were invited to use the NSF CC* Cyberteam SWEETER Slack workspace [1]. In addition to offering course-related resources, such as slide decks and Jupyter Notebooks, these sessions were recorded and offered as YouTube videos. These recordings are available free-of-charge via the Texas A\&M HPRC YouTube channel. Closed captioning was included on each video, and the videos met Texas A\&M's requirements for the Americans with Disabilities Act. 


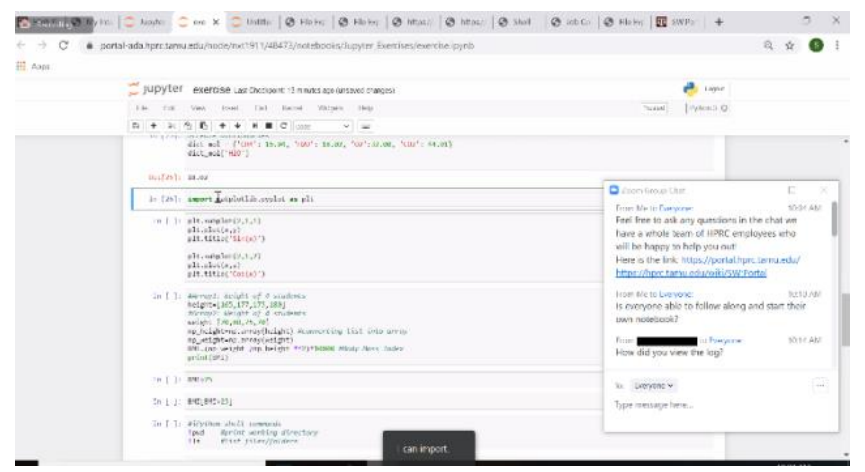

Figure 1. A screenshot of the teaching interface. Here the peer-instructor is working on a Jupyter Notebook while the peer-moderator encourages and supports a parallel discussion in the Zoom chat window.

\section{RESULTS AND FUTURE WORK}

Here, we briefly describe the results from our online short course and Primer programs. A complete list of our training activities is available on the HPRC website.

\subsection{Short Courses Offered}

In Spring 2020, we completed our planned bouquet of Spring 2020 short courses in an online-only format using the Zoom platform. This was followed by an online series of short courses on Quantum Mechanics offered in Summer 2020. In Fall 2020, the shortcourses returned to our offering. The move to an online-only platform did not impact the number of participants registering for our short courses. Registration and participation in the Spring 2020 short courses mimicked that of previous semesters, when the courses were taught in the hybrid in-person and online format. Since all interactions were now via Zoom, we noticed that the interactions between the instructor and the attendees were much more limited. This was a marked change from the in-person interactions between the instructor and the participants, and it has been ascribed to variety of factors, ranging from technology limitations, poor internet connections, participant hesitation to speak out in front of a larger audience, a reluctance to enter questions into chat forums, competing online distractions, and a lack of engagement with instructor or course materials.

\subsection{Primer Courses Offered}

The Primer courses were launched in late Spring 2020. The Primers were advertised and managed using our regular broadcast email, and registration and content was managed via our website and Google Forms. To our pleasant surprise, and perhaps an indicator of the rising demand for research computing, all course offerings were selected, and we rapidly reached the minimum threshold of five learners for each Primer course. Primers were offered on introductory topics related to Linux, CLI, Cluster Usage, scheduler usage (SLURM and IBM Spectrum Scale LSF), using the OpenOnDemand Portal, Data Management Practices, and using Jupyter notebooks. A listing of all Primer courses offered in 2020, and the number of students registered per course are presented in Table 1.

For the purposes of brevity and maintaing clarity, Primer courses are grouped in terms of Operating Systems (Linux), Technology (Jupyter Notebooks and Data Management Practices), Schedulers (LSF and SLURM), and Clusters (Ada and Terra) in this manuscript. The portal refers to Texas A\&M HPRC's implementation of the OpenOnDemand portal developed by Ohio Supercomputer Center. In all, 15 Primers were offered.
Table 1. List of Primer courses, and the number of registered attendees for each session, from Spring 2020 to Fall 2020. The primers are listed in the order in which they were presented.

\begin{tabular}{|c|c|c|}
\hline Semester & Courses & Registered \\
\hline \multirow{6}{*}{$\begin{array}{l}\text { Spring } \\
2020\end{array}$} & $\begin{array}{l}\text { Introduction to Linux w/ } \\
\text { MobaXterm }\end{array}$ & 126 \\
\hline & Introduction to the Ada Cluster & 98 \\
\hline & LSF: Job Scheduling & 44 \\
\hline & Introduction to the Terra Cluster & 92 \\
\hline & SLURM: Job Scheduling & 27 \\
\hline & Data Management Practices & 96 \\
\hline \multirow{9}{*}{$\begin{array}{c}\text { Summer } \\
2020\end{array}$} & $\begin{array}{c}\text { Introduction to HPRC - Clusters, } \\
\text { Duo, VPN }\end{array}$ & 63 \\
\hline & Jupyter Notebooks on the Portal & 59 \\
\hline & $\begin{array}{c}\text { Introduction to Linux w/ } \\
\text { MobaXterm }\end{array}$ & 40 \\
\hline & Introduction to Linux w/ Portal & 39 \\
\hline & Introduction to the Ada Cluster & 42 \\
\hline & LSF Job Scheduler & 44 \\
\hline & Introduction to the Terra Cluster & 43 \\
\hline & SLURM Job Scheduler & 69 \\
\hline & Data Management Practices & 91 \\
\hline \multirow{7}{*}{ Fall 2020} & Introduction to Linux w/ Portal & 71 \\
\hline & Introduction to the Terra Cluster & 54 \\
\hline & Introduction to the Ada Cluster & 64 \\
\hline & Data Management Practices & 70 \\
\hline & SLURM: Job Scheduling & 53 \\
\hline & LSF: Job Scheduling & 55 \\
\hline & Jupyter Notebooks on the Portal & 67 \\
\hline
\end{tabular}

On average, about 55 participants registered for each Primer course. Due to the unique registration format, registered participant counts include those who showed interest in the topic and didn't have a preference for the day on which the course was offered. It is noteworthy that since new graduate student enrollment is typically highest in Fall semester, we see typically see a drop-off in participation in our Introductory short courses in the Spring semester. The registration numbers for Spring reflect enthusiasm for both computing and the new learning format at that time. In response to the continued demand for quick online programs, the Primers were offered a second time in Summer of 2020.

Summer attendance in the Primers series was encouraged by the summer research learning programs such as the Online Research Experiences for Undergraduates program at Texas A\&M. A slight drop in registrations was observed. In Fall 2020, we continued to work in an online-only setting. As such, the Primers program ran in parallel with the Short course program. Figure 2 shows that a greater number of learners registered for the Primers in the Spring, Summer, and Fall semesters of 2020, as compared to those registered for similarly-themed Introductory short courses that were offered in the hybrid in-person and online format in Fall 2019. Perhaps a testament to the success of this online-only format is the continuing participation in Fall 2020. 


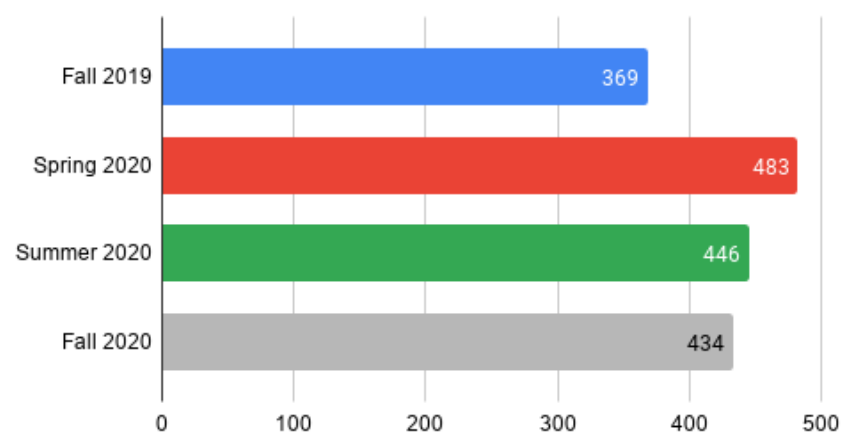

Figure 2. Total number of participants registered for Primer courses in Spring (red), Summer (green), and Fall (gray) semesters of 2020 . For comparison, we show the number of students who participate in the 2.5-hour-long course in Fall 2019 (blue).

\subsection{Participation Trends}

Primers maintained student interest in all the major categories, as shown in Figure 3. Consistent with the class of new researchers using our facilities, we saw increased participation in courses related to using the campus clusters and interactive technologies like Jupyter notebooks. Polling data collected during the registration process found that nearly all participants voted for all the topics. As such, beyond telling us that the pre-selected topics were of interest, crowd-sourcing did not provide clear guidance on what sub-topics to teach. Our SWEETER Slack workspace offers a rich collaborative space that connects over 470 researchers from several countries. It includes several public and private channels related to research computing and software usage. We also find that while several learners joined the SWEETER slack, most discussions took place on private topic-specific channels rather than on a public channel. As the courses progressed we learned that while the interactive sessions were scheduled for 10 minutes, they may carry on for up to 30 minutes after a primer course. As such, we assume that the Primers filled a significant knowledge gap for researchers new to research computing clusters environments.

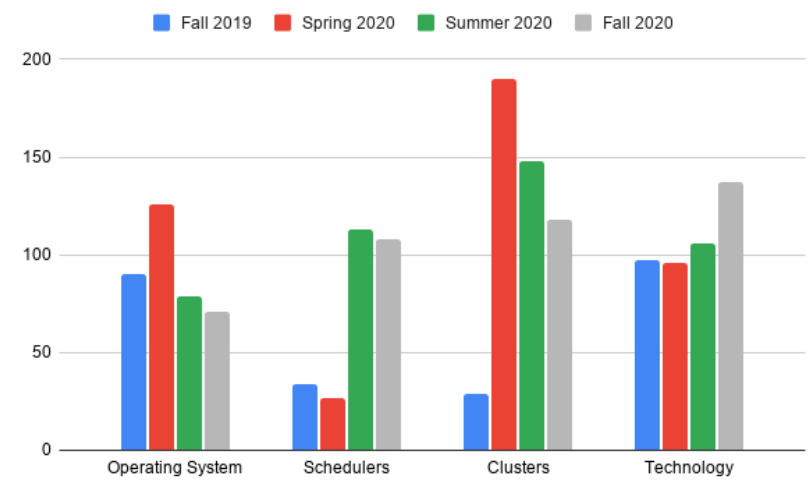

Figure 3. Participation in Primers in the Spring, Summer, and Fall semsters of 2020. Participation in the 2.5-hour version of short courses in Fall 2019 is shown as a benchmark for when longer sessions were offered on these topics.
Texas A\&M HPRC supports users from several fields of science. Figure 4 shows the participation of learners from various colleges representing different fields of science. We find that the courses maintained the cross-disciplinary appeal that was observed in our short courses program in Fall 2019. Increased participation from Engineering disciplines was observed. This possibly ties into the increased use of computing in engineering research, students wanting to learn new research skills during the downtime brought about by the COVID-19-implied norm, and possibly because learners were now able to tune into an online course, rather than travel to a classroom on the other end of campus.

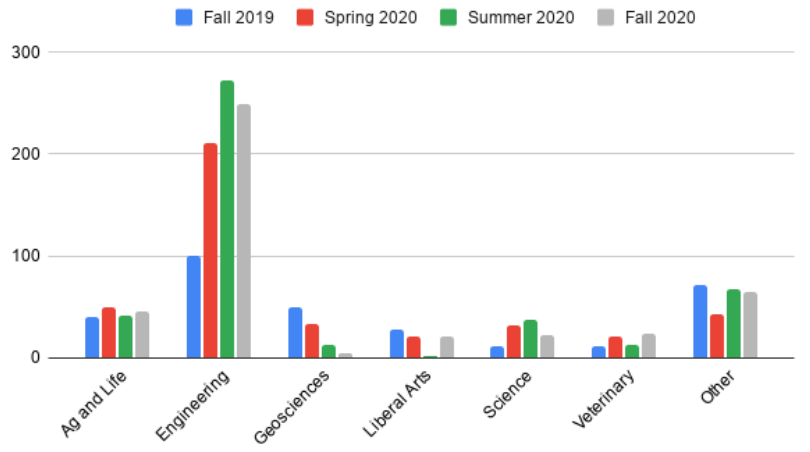

Figure 4. Participants per college for Spring, Summer, and Fall semesters of 2020. For comparative purposes, we include data from Fall 2019.

\subsection{Learner Persistence}

Tracking how students approach the topics offered by the program and learner persistence are key considerations for improvements in future iterations. For each of these live-streamed Primer courses, persistence was tracked along two lines of enquiry. First we observed how long a participant remained on during a course, and next we saw how many Primers courses were attend by a learner.

Here, we report on our findings for the Primers offered in the 2020 calendar year. As described above, our original target participation for our courses was five participants per course. In order to track persistence, i.e. what percentage of students complete the session, across a Primer course, we observed how long a participant remained on the Zoom session. The data from the calendar year is shown in Figure 5. Here we find a slight drop-off in the first 15 minutes. The majority of learners (greater than 60\%) complete the hour-long exercises and stay for the Open Mic session. We hypothesize that the early drop rate could be reflective of various factors. Learners could have realized that they have either signed up for the wrong class, that the class materials and course recording are available for later viewing, that the materials do not meet with their expectations, or perhaps they have unstable Internet connectivity. We find that as we got into the Summer and Fall semesters, more students remained until the conclusion of the course. We surmise this is because learners are becoming more familiar with the platform and adjusting their expectations. Noting that this metric may be an indicator of the popularity of the Open Mic session that happens after the Primer, we point out that participation in these sessions varies depending on the topic and the audience on a given day. 


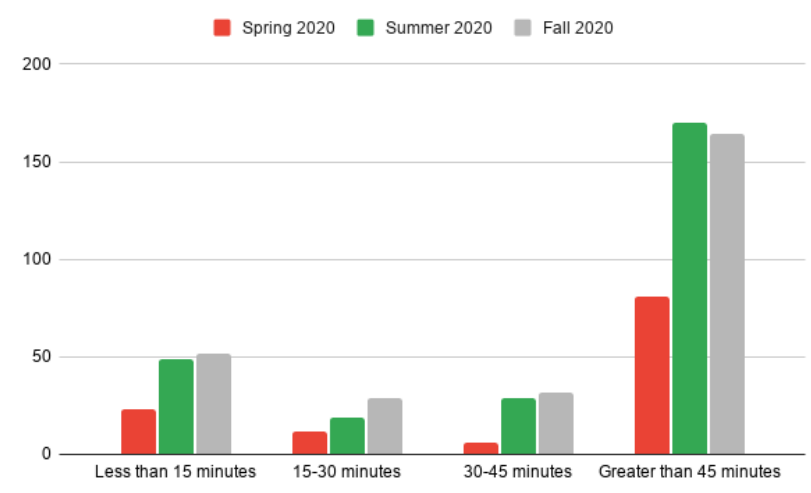

Figure 5. Learner persistence in each Primer session. Number of minutes spent by Zoom attendees ( $\mathrm{Y}$-axis) in Spring, Summer, and Fall Semesters of 2020.

Learner participation in the program was tracked across each semester, and across multiple semesters. As described previously, the Primers are geared to give relatively quick bursts of information and are not tiered for a longer or sustained learning effort. As shown in Figure 6 (a), we find that consistent with our intended goals, $45 \%$ of learners attended a primer on a given topic, and $43 \%$ of learners continued to participate in two or more classes. Figure 6 (b) shows the distribution of learning across semesters. We find that consistent with the goals of the program, the overwhelming number of learners attend Primers in a single semester. A small percentage of learners availed of the primers across two semesters.

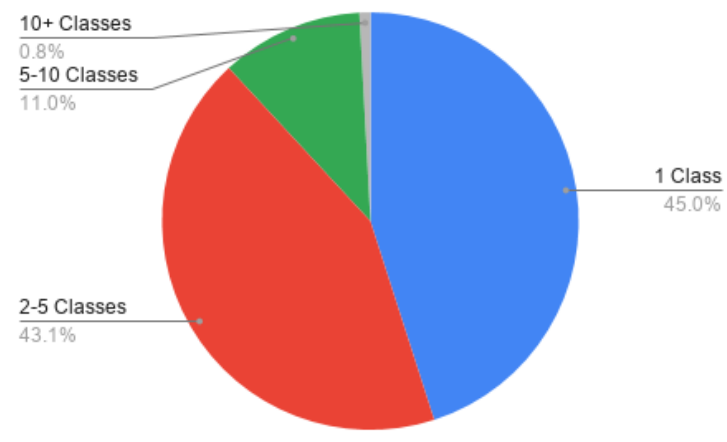

Figure 6 (a). Total courses registered per learner across the Fall, Summer, and Spring 2020 semesters.

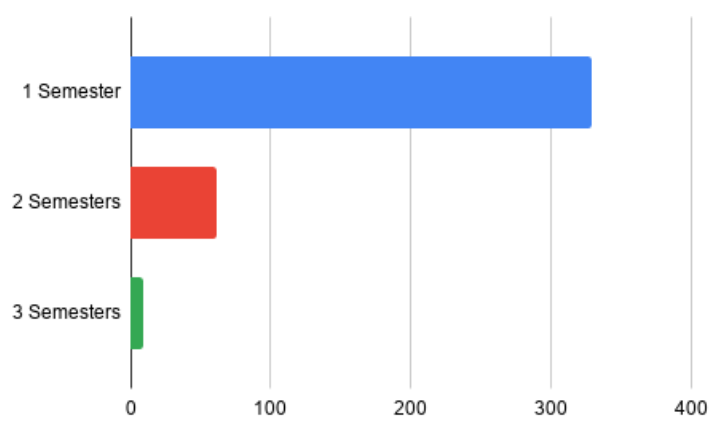

Figure 6 (b). Number of learners registered for multiple semesters across the Fall, Summer, and Spring 2020 semesters.

\subsection{Staged Curricular Materials}

We have continued to stage our teaching materials and exercises on online platforms. Our website [1] hosts a collection of our training materials. These materials are updated by the instructors each time the Primers are offered. For the Fall 2020 semester, we find that the Primer course slide decks and notebooks for the Primers were downloaded 517 times by individuals and $\sim 30$ times by bot services. Details of downloads per course and thematic areas are shown in Table 2, and the breakdown across thematic areas is shown in Figure 7. Consistent with Primer registration, we find that cluster usage dominated among these categories.

Table 2. List of Primer and short videos offered on the Texas A\&M YouTube channel and associated views.

\begin{tabular}{|c|c|c|}
\hline Type & Courses & Views \\
\hline \multirow{10}{*}{$\begin{array}{l}\text { Introductory } \\
\text { Videos } \\
\text { (5 minutes } \\
\text { or less) }\end{array}$} & What is Texas A\&M HPRC? & 174 \\
\hline & Applying for Accounts & 141 \\
\hline & Cluster Access using SSH & 113 \\
\hline & Accessing Cluster from Windows & 33 \\
\hline & File Management on Clusters & 99 \\
\hline & Managing Allocations & 122 \\
\hline & Modules System & 56 \\
\hline & Submitting a Job using LSF & 162 \\
\hline & Submitting a Job using SLURM & 23 \\
\hline & $\begin{array}{c}\text { Submitting a Job File using } \\
\text { Tamubatch }\end{array}$ & 100 \\
\hline \multirow{9}{*}{$\begin{array}{l}\text { Primers } \\
\text { (45 to } 60 \\
\text { minutes) }\end{array}$} & $\begin{array}{c}\text { Introduction to HPRC - Clusters, } \\
\text { Duo, VPN }\end{array}$ & 77 \\
\hline & Jupyter Notebooks on the Portal & 65 \\
\hline & Introduction to Linux & 13 \\
\hline & Introduction to Linux on a portal & 96 \\
\hline & Using the Ada Cluster & 173 \\
\hline & LSF Job Scheduler & 34 \\
\hline & Using the Terra Cluster & 93 \\
\hline & SLURM Job Scheduler & 64 \\
\hline & Data Management Practices & 34 \\
\hline
\end{tabular}

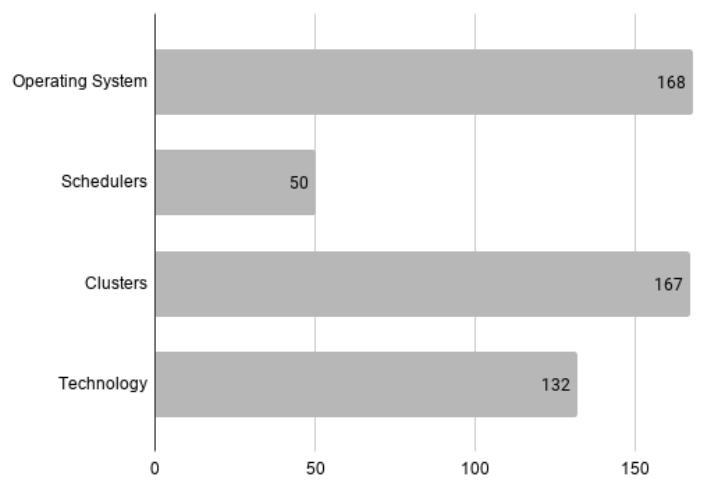

200

Figure 7. Distribution of downloaded primer course materials by themes for Fall 2020. 


\subsection{Efficacy of Online Videos}

Recordings of the hour-long Primer sessions are offered on the Texas A\&M HPRC YouTube Channel. The channel hosts 34 instructional videos in short (five-minute), medium (45-to-60minute), and long (two-hour) durations that have amassed over 2,500 views. All videos are indexed (bookmarked) and checked for the accuracy of the closed captioning. Since its launch in late April 2020, the channel has gained over 139 subscribers as of November 2020. Complementing the Primer videos are short (less than 5minute) videos on topics such as how to access the HPRC clusters. An analysis of the videos shows that learners are more likely to gravitate toward shorter videos as opposed to more detailed videos. A detailed breakdown of viewership statistics is presented in Table 3. Viewership and subscription data were collected at the time of writing this manuscript to show the differential impact of vlength versus usage.

Table 3. List of course material downloaded by individuals for HPRC Primers offered in Fall 2020.

\begin{tabular}{|c|c|}
\hline Courses & Learner Downloads \\
\hline Introduction to Linux & 168 \\
\hline Introduction to the Ada Cluster & 83 \\
\hline Introduction to the Terra Cluster & 52 \\
\hline Data Management Practices & 62 \\
\hline Introduction to HPRC & 32 \\
\hline Jupyter Notebooks on OOD & 70 \\
\hline LSF Job Scheduler & 10 \\
\hline SLURM Job Scheduler & 40 \\
\hline
\end{tabular}

In keeping with the philosophy of Open Science, all materials are available free-of-charge for use and adoption to the larger research computing community. The encouraging viewership of YouTube videos by the Research community, while heartening, revealed that a significant portion of our viewership came from outside the United States. Approximately a third of our viewers used the closed captioning service on these videos. Figure 8 shows the viewership trends for the shorter 5-minute videos versus the longer Primerrecorded (1-hour) videos on YouTube. The total viewership minutes per category, calculated by multiplying the total viewership of a video by the duration of the video, remains approximately the same in each category. As such, one may hypothesize that while shorter videos are more likely to reach out to a broader audience, the longer one-hour videos serve an important purpose by helping learners who are interested in a slightly deeper dive into the topic. We once again note that courses on cluster usage get the most viewership.

\section{CONCLUSIONS}

The data collected as part of this study show that the Primer format could be a suitable pedagogical approach that enhances learner engagement, makes the materials more relatable, and leverages peer-learning and peer-led-discussion approaches while scaling back on staff time. The courses in conjunction with the online communities, pre-staged materials, and online videos showed increased participation from learners and were a better fit for an online-only educational platform. It is heartening to note that despite these viewership of materials on YouTube and availability of course materials on our website, the Primers consistently engaged new learners, and participation remained high in the Summer and Fall semesters of 2020.

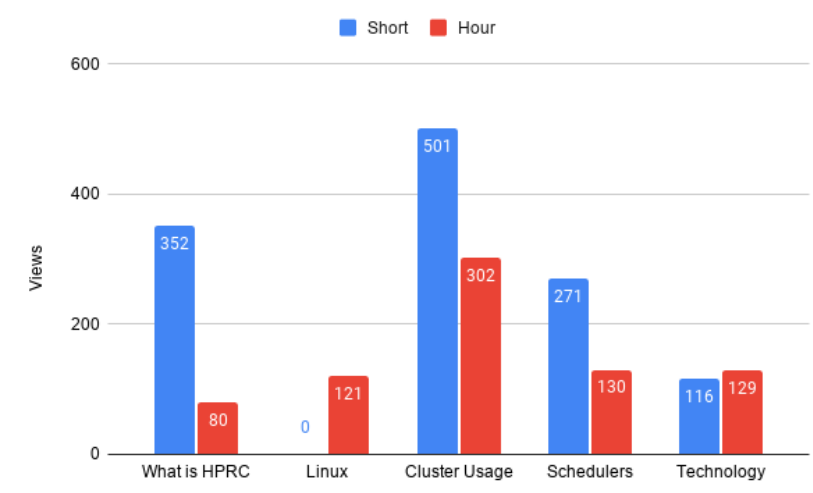

Figure 8. Distribution of short video ( $<5$ minute) versus longer (1-hour) videos in topic areas.

\section{CHALLENGES AND FUTURE WORK}

While the Primer course format is better suited for an exclusivelyonline instruction-dependent world, challenges remain. Getting participants to complete evaluations that gauge the effectiveness of our program is a challenge. While we traditionally had over $70 \%$ of participants respond to surveys, we saw responses drop to $10 \%$ upon switching to Google Forms. We have since switched to polling participants over Zoom and observe upwards of 50\% participation. We note that Zoom is a limited medium compared to the richness of Google Forms. Our questions today are limited to:

1. Did you attend this course for research, personal, and/or class needs?

2. Did the course meet your objectives?

3. Would you like future courses to be more generalized, specialized, or both?

Moving to online-only usage of resources encouraged us to explore mechanisms to improve and scale our training operations. The last couple of semesters have shown us the strengths of adopting an online-only approach. As a nation, we have collectively observed that training over online resources has its own share of questions related to access, inclusivity, equity, and diversity [6]. While we celebrate the expanded reach enabled by offering training over the Internet, we sadly realize that students with limited access to technology and reliable Internet connectivity are in danger of being left behind. Today, HPRC is experimenting with a new online pedagogical approach, called the "technology labs" [1]. These labs are geared toward placing the participants in a real-world scenario on entry. At the time of writing this manuscript, it is hard not to acknowledge that we stand at the crux of a "twindemic" that could well progress the remote-only settings to the Summer of 2021 or beyond. Indeed, at Texas A\&M University, the Spring 2021 semester has been adjusted. Based on the usage characteristics, we plan to offer these courses in an online setting into the foreseeable future.

\section{SUPPORTING INFORMATION}

All training materials used in this study are available to the community via the Texas A\&M HPRC website at https://www.hprc.tamu.edu/training. Videos and course recordings may be accessed via the Texas A\&M HPRC channel on YouTube. The community is invited to join the SWEETER Slack workspace at https://hprc.tamu.edu/sweeter. Surveys and review exercises that will be developed as part of this longitudinal study may be requested from the author. Please send us feedback about your adoption experience via an email to help@hprc.tamu.edu. 


\section{ACKNOWLEDGEMENTS}

The authors thank the staff at Texas A\&M HPRC for assisting with the research related to this study. We gratefully acknowledge support from the National Science Foundation (NSF). We thank the NSF for award \#1649062, "NSF Workshop: Broadening Participation in Chemical and Biological Computing at the Early Undergraduate Level," award \#1730695, "NSF CyberTraining: CIP: CiSE-ProS: Cyberinfrastructure Security Education for Professionals and Students," award \#2019136, "NSF CC* BRICCs: Building Research Innovation at Community Colleges," and award \# 1925764, "NSF CC* SWEETER: South West Expertise in Expanding Training Education and Research."

\section{REFERENCES}

[1] Texas A\&M High Performance Research Computing website. URL: https://hprc.tamu.edu

[2] D. K. Chakravorty, M. Pennings, H. Liu, Z. Wei, D. M. Rodriguez, Levi T. Jordan, D. F. McMullen, N. Ghaffari, and S. D. Le. "Effectively Extending Computational Training Using Informal Means at Larger Institutions," Journal of Computational Science Education 2018, 40-47 DOI: 10.22369/issn.2153-4136/10/1/7

[3] D. K. Chakravorty, M. Pennings, H. Liu, Z. Wei, D. M. Rodriguez, L. T. Jordan, D.F. McMullen, N. Ghaffari, S. D. Le, D. Rodriquez, C. Buchanan, and N. Gober. "Evaluating Active Learning Approaches for Teaching Intermediate Programming at an Early Undergraduate Level," Journal of Computational Science Education 2018, 61-66 DOI 10.22369/issn.2153-4136/10/1/10

[4] D. K. Chakravorty, D. F. McMullen, N. Gober, J. H. Seo, M. Bruner, and A. Payne. "Using Virtual Reality to Enforce Principles of Cybersecurity," Journal of Computational Science Education 2018, 81-87 DOI 10.22369/issn.21534136/10/1/13

[5] D. K. Chakravorty, M. Pennings, H. Liu, X. Thomas, D. M. Rodriguez, and L.M. Perez. "Incorporating Complexity in Computing Camps for High School Students - A Report on the Summer Computing Academy Program at Texas A\&M University," Journal of Computational Science Education 2020, 11, 1 12-20 DOI https://doi.org/10.22369/issn.2153$4136 / 11 / 1 / 3$
[6] D. K. Chakravorty, M. T. Pham "Evaluating the Effectiveness of an Online Learning Platform in Transitioning from High Performance Computing to a Commercial Cloud Computing Environment," Journal of Computational Science Education 2020, 11, 1, 93-99.

[7] Diverse Education News: Impact on Disadvantaged students https://diverseeducation.com/article/189597/

[8] K. Saichaie, D. C. Brooks, P. Long, R. Smith, R. Holeton, C. Meyers, A. Finkelstein, S. Dugdale, "7 Things You Should Know About Research on Active Learning Classrooms," in ELI 7 Things You Should Know, Educause Learning Initiative (ELI), 2017.

[9] P. Baepler, J. D. Walker, D. C. Brooks, K. Saichaie, C. I. Petersen, B. A. Cohen, "A Guide to Teaching in the Active Learning Classroom: History, Research, and Practice," Stylus Publishing, ISBN-13: 978-1620363003, 2016.

[10] Student-Centered Active Learning Environment with Upside-down Pedagogies: http://scaleup.ncsu.edu/

[11] M. Prince, "Does active learning work? a review of the research," Journal of engineering education, vol. 93, no. 3, pp. 223-231, 2004.

[12] J. Parsons and L. Taylor, "Improving student engagement," Current issues in education, vol. 14, no. 1, 2011.

[13] Larkin, M. (2002). Using scaffolded instruction to optimize learning. http://www.vtaide.com/png/ERIC/Scaffolding.htm

\section{A. REPRODUCIBILTY INDEX}

All training materials are available via our website at https://hprc.tamu.edu. Videos are available free-of-cost via the Texas A\&M HPRC channel on YouTube. Surveys, analytics for Slack and YouTube, and review exercises that will be developed as part of this longitudinal study may be requested from the author. Please send us feedback about your adoption experience, questions, and requests to join our training Slack (SWEETER Slack) via an email to help@hprc.tamu.edu. 